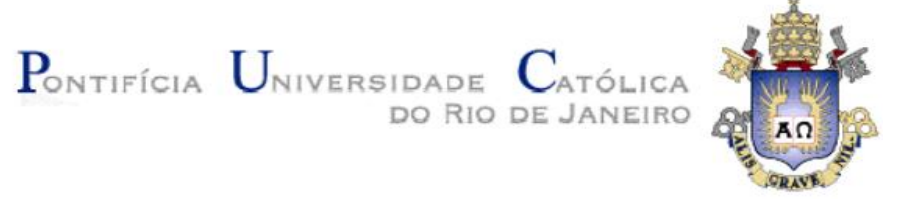

Fagner Henrique Guedes Neves

Conhecimento, Escola e Cultura/s:

Ensino de Sociologia e Educação Intercultural

Dissertação de Mestrado

Dissertação apresentada ao Programa de Pós-graduação em Educação da PUC-Rio como requisito parcial para obtenção do título de Mestre em Educação.

Orientadora: Prof ${ }^{a}$ Vera Maria Ferrão Candau

Rio de Janeiro

Março de 2014 
Fagner Henrique Guedes Neves

\title{
Conhecimento, Escola e Cultura/s: Ensino de Sociologia e Educação Intercultural
}

\begin{abstract}
Dissertação apresentada como requisito parcial para obtenção do título de Mestre em Educação do grau de Mestre pelo Programa de Pós-graduação em Educação do Centro de Teologia e Ciências Humanas da PUC-Rio como requisito parcial. Aprovada pela Comissão Examinadora abaixo assinada.
\end{abstract}

\author{
Profạ. Vera Maria Ferrão Candau \\ Orientadora \\ Departamento de Educação - PUC-Rio \\ Profa. Isabel Alice Oswald Monteiro Lélis \\ Departamento de Educação - PUC-Rio \\ Prof. Paulo Pires de Queiroz \\ UFF \\ Prof?. DENISE BERRUEZO PORTINARI \\ PUC-Rio
}

Coordenadora Setorial do Centro de Teologia e Ciências Humanas

Rio de Janeiro, 24 de Março de 2014 
Todos os direitos reservados. É proibida a reprodução total ou parcial sem autorização da Universidade, do Autor e da Orientadora.

\section{Fagner Henrique Guedes Neves}

Graduado em Ciências Sociais e Especialista em Ensino de História e Ciências Sociais pela Universidade Federal Fluminense - UFF. Servidor Público lotado na UFF.

Ficha Catalográfica

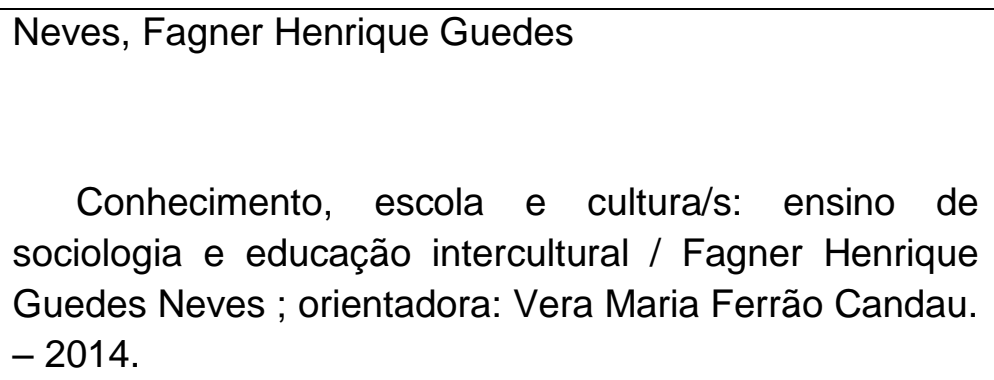

Conhecimento, escola e cultura/s: ensino de sociologia e educação intercultural / Fagner Henrique Guedes Neves ; orientadora: Vera Maria Ferrão Candau. $-2014$.

144 f. il. (color.) ; $30 \mathrm{~cm}$

Dissertação (mestrado)-Pontifícia Universidade Católica do Rio de Janeiro, Departamento de Educação, 2014.

Inclui bibliografia

1. Educação - Teses. 2. Conhecimento. 3. Escola. 4. Ensino de sociologia. 5. Educação intercultural. I. Candau, Vera Maria Ferrão. II. Pontifícia Universidade Católica do Rio de Janeiro. Departamento de Educação. III. Título. 


\section{Agradecimentos}

- Nenhuma seção de agradecimento que eu faça pode começar sem minha menção a meu Eterno Mestre: Deus, especificamente o Deus das Escrituras Judaicocristãs. Sei que tal proposição se choca aos relativismos típicos da modernidade tardia e ao ethos cientificista agnóstico, típico do imaginário social da Academia. Não me intimido, porém, com possíveis constrangimentos movidos por estas forças. Afirmarei neste espaço que, mesmo em meio a meus estudos e experiências acadêmicas, tenho Fé nesse Deus e desta retiro forças para meu viver. Neste sentido, cabe a Deus o meu primeiro e inegável agradecimento.

- Agradeço imensamente à Prof ${ }^{a}$ Vera Candau pelas preciosas orientações concedidas durante a realização deste trabalho. Obrigado também pelos estudos e discussões proporcionados nos âmbitos da disciplina "Metodologia Didática" e do Grupo de Estudos em Cotidiano, Educação e Culturas - GECEC-PUC-Rio.

- Muito obrigado ao Prof. Paulo Pires de Queiroz [FEUFF] pelas significativas oportunidades de exposição trabalhos meus nestes dois anos. Sou bastante grato.

- Agradeço a todos/as os/as Professores do PPGE/PUC-Rio com os quais tive oportunidade de interagir nestes dois anos: Isabel Lélis, Cynthia Paes de Carvalho, Alícia Bonamino, Marcelo Andrade, Ralph Ings Bannell, Rosália Duarte e Zaia Brandão. Obrigado por suas aulas e/ou avaliações, que certamente colaboraram ao meu desenvolvimento intelectual.

- Agradeço aos colegas de classe e grupo pelas interações e debates.

- Meus agradecimentos também se destinam aos/às funcionários/as do Departamento de Educação da PUC-Rio por seus atenciosos e prestativos atendimentos.

- Muito obrigado à Vice-Reitoria de Assuntos Comunitários da PUC-Rio pela quitação das mensalidades referentes a este curso.

- Por fim, não posso esquecer do apoio de meus familiares durante o período deste curso: minha Mãe, Maria Edna; meu Pai, José Henrique; e minha irmã, Andrea. Muito Obrigado por participarem de minha trajetória há quase vinte e nove anos. 


\section{Resumo}

Neves, Fagner Henrique Guedes; Candau, Vera Maria Ferrão (Orientadora). Conhecimento, Escola e Cultura/s: Ensino de Sociologia e Educação Intercultural. Rio de Janeiro, 2014, 144p. Dissertação de Mestrado Departamento de Educação, Pontifícia Universidade Católica do Rio de Janeiro.

Este trabalho situa-se na confluência entre conhecimento, educação escolar, ensino de Sociologia e interculturalidade, um diálogo pouco explorado pela pesquisa educacional brasileira. Tendo como referenciais os estudos interculturais de Boaventura de Sousa Santos, Vera Maria Candau e Antônio Flávio Moreira, busca-se discutir como os professores de Sociologia lotados na escola básica compreendem as possibilidades de debates entre o saber sociológico escolar e a educação intercultural. Neste empreendimento, dois objetivos são visados: (1) identificar as representações de professores de Sociologia de escolas públicas de Niterói sobre as relações entre o conhecimento sociológico escolar vigente na escola básica brasileira e a proposta da educação intercultural e a (2) problematizar possibilidades de construção de currículos escolares sociológicos interculturalmente orientados no contexto de escolas da rede pública. Para tanto, foram desenvolvidas entrevistas individuais semiestruturadas com onze sujeitos licenciados em Ciências Sociais e atuantes no magistério estadual de Sociologia há pelo menos dois anos. Foram também analisados documentos curriculares oficiais voltados ao ensino médio e à disciplina de Sociologia. Mediante a articulação entre os dados obtidos através desses procedimentos e os referenciais teórico-conceituais enunciados, foi possível obter significativos achados. A despeito de diversas proposições favoráveis à educação intercultural nos documentos curriculares analisados, esta ainda é escassamente promovida na seleção de conteúdos e no desenvolvimento de práticas pedagógicas no ensino básico de Sociologia, conforme relatam os sujeitos da pesquisa. Nesse cenário, a construção intercultural do conhecimento sociológico escolar é uma meta a se cumprir, repleta de desafios a serem enfrentados pelo sistema escolar e os educadores.

\section{Palavras-chave}

Conhecimento; Escola; Ensino de Sociologia; Educação Intercultural. 


\section{Abstract}

Neves, Fagner Henrique Guedes; Candau, Vera Maria Ferrão (Advisor). Knowledge, School and Culture: Sociology Teaching and Intercultural Education. Rio de Janeiro, 2014, 144p. MSc. Dissertation - Departamento de Educação, Pontifícia Universidade Católica do Rio de Janeiro.

This work approaches an unusual discussion in the Brazilian educational research, involving knowledge, school education, Sociology teaching, and interculturalism. Specifically, the work focuses the public high-school Sociology teachers' opinions about the dialogues between the Sociology knowledge and the project of the intercultural education, considering the conceptions from Boaventura de Sousa Santos, Vera Maria Candau, and Antônio Flávio Moreira. There are two research goals: (1) to identify the high-school Sociology-teachers' representations about the Sociology knowledge which is normally taught at the Brazilian Schools and its relationships with the intercultural education; (2) to propose some possibilities of creating Sociology public high-schools curricula under the intercultural concepts. Semi-structured interviews with eleven Social Sciences licensed-teachers who have been working at public high-schools in Niterói City (State of Rio de Janeiro) for at least two years were made. In addition, the official curricula documents concerning Sociology teaching and high-school education were analyzed. Linking the achieved data with the theoretical references, some important results were found. Although the analyzed documents point many propositions around the intercultural constitution of the Sociology teaching, content choices and the pedagogical practices are not usually affected by the intercultural education. At this scenery, building a high-school Sociology teaching under intercultural ideas remains as a non-reached goal, which is plenty of challenges to be faced by the educators.

\section{Keywords}

Knowledge; School; Sociology Teaching; Intercultural Education. 


\section{Sumário}

1. Introdução

2. A construção histórica de um objeto: o Ensino de Sociologia na política e na pesquisa educacional brasileira

2.1. De 1882 a 2013: uma retrospectiva do ensino de Sociologia na política educacional brasileira

2.2. Aspectos quantitativos e qualitativos da pesquisa sobre ensino de Sociologia no país

3. Educação Intercultural e Conhecimento Escolar

3.1. Sociologia das Ausências e Sociologia das Emergências e a perspectiva intercultural dos estudos de Boaventura de Sousa Santos

3.2. O conhecimento escolar como um campo intercultural

4. Materiais e Métodos de Pesquisa

4.1. Natureza da Pesquisa

4.2. Campo da Pesquisa

4.3. Sujeitos da Pesquisa

56

4.4. Estratégias Metodológicas

58

4.4.1. A Técnica de Triangulação de Dados 59

4.4.2. Entrevistas individuais semiestruturadas 60

4.4.3. Análise documental

66

5. A construção intercultural do conhecimento sociológico escolar: proposições docentes e desafios a enfrentar

5.1. Quem são os sujeitos da pesquisa?

5.2. Os depoimentos dos sujeitos da pesquisa

5.2.1 Iniciando as entrevistas: relatos dos/as professores/as sobre sua formação profissional docente e os contextos em que atuam

5.2.2 Ensino de Sociologia e Educação Intercultural: o que pensam os/as professores/as?

5.3. A construção intercultural do conhecimento sociológico escolar: seus fundamentos e desafios característicos 
6. Considerações Finais

7. Referências Bibliográficas

Anexos 


\section{Lista de Quadros}

Quadro 1 - Ensino escolar de Sociologia no período entre 1882 e 1925

Quadro 2 - Ensino escolar de Sociologia no período entre 1925 e 1942

Quadro 3 - Ensino escolar de Sociologia no período entre 1942 e 1982

Quadro 4 - Ensino escolar de Sociologia no período entre 1983 e 2008

Quadro 5 - Encontros sobre ensino de Sociologia realizados entre 2007 e 2013

Quadro 6 - As cinco monoculturas identificadas por Santos 43

Quadro 7 - As cinco ecologias propostas por Santos

Quadro 8 - Os critérios de análise documental preliminar propostos por Cellard

Quadro 9 - Análise preliminar das DCNEM 68

Quadro 10 - Análise preliminar dos PCNEM 69

Quadro 11 - Análise preliminar das OCNEM 70

Quadro 12 - Análise preliminar do Currículo Mínimo de 


\section{Lista de Tabelas}

Tabela 1 - Produção sobre Ensino de Sociologia nos últimos dez anos

Tabela 2 - Marcadores de diferença e identidade cultural mencionados pelos/as entrevistados/as

Tabela 3 - Conflitos mencionados pelos/as entrevistados/as, referentes a marcadores de diferença e identidade cultural 


\section{Lista de Abreviaturas e Siglas}

Art. - Artigo

ASPERJ - Associação dos Sociologos do Rio de Janeiro

BID - Banco Interamericano de Desenvolvimento

CAPES - Comissão para o Aperfeiçoamento de Pessoal de Ensino Superior

CEPEBA - Centro de Estudos e Pesquisas da Baixada Fluminense

Cf. - Conferir

CNE - Conselho Nacional de Educação

DCNEM - Diretrizes Curriculares Nacionais para o Ensino Médio

EJA - Educação de Jovens e Adultos

ENESEB - Encontro Nacional de Ensino de Sociologia da SBS

ENSOC - Encontro Estadual sobre o Ensino de Sociologia do Rio de Janeiro

FASE - Federação de Órgãos para Assistência Social e Educacional

GECEC - Grupo de Estudos em Cotidiano, Educação e Culturas do Departamento

de Educação da PUC-Rio

IBASE - Instituto Brasileiro de Análises Sociais e Econômicas

LDB - Lei de Diretrizes e Bases da Educação

MEC - Ministério da Educação

$\mathrm{n}^{\mathrm{o}}$ - Número

p. - Página

PCNEM - Parâmetros Curriculares Nacionais para o Ensino Médio

PDT - Partido Democrata Trabalhista

PT - Partido dos Trabalhadores

PL - Projeto de Lei

PR - Estado do Paraná

PNLD - Programa Nacional do Livro Didático

PUC-Rio - Pontifícia Universidade Católica do Rio de Janeiro

OCNEM - Orientações Curriculares Nacionais para o Ensino Médio

ONU - Organização das Nações Unidas

RJ - Estado do Rio de Janeiro

SBS - Sociedade Brasileira de Sociologia

SEEDUC/RJ - Secretaria de Estado da Educação do Rio de Janeiro 
TICs - Tecnologias da Informação e da Comunicação

UERJ - Universidade do Estado do Rio de Janeiro

UFC - Universidade Federal do Ceará

UFF - Universidade Federal Fluminense

UFPR - Universidade Federal do Paraná

UFRJ - Universidade Federal do Rio de Janeiro

UFRN - Universidade Federal do Rio Grande do Norte

USP - Universidade de São Paulo 